\title{
Cystic Adenoid Carcinomas in Ear, Nose and Throat: Unusual Localizations and a Literature Review
}

\author{
F. Hadid, M. Hakimi, O. Oulghoul, O. Benhommad, Y. Rochdi, and A. Raji
}

\section{ABSTRACT}

Introduction: adenoid cystic carcinoma account $1 \%$ for all cancers of the ear nose and throat. Despite slow growth, their recurrence is frequent. This study concerns 5 cases of cystic adenoid carcinoma with atypical ear nose and throat locations (outside the main salivary glands).

Materials and methods: Our work is a study of 5 cases of cylindromes with atypical localizations collected at the ear nose throat and cervico-facial surgery department of Mohamed IV university hospital of Marrakesh: 1 case located at the external auditory meatus, 1 case at the infratemporal fossa, 1 case on the inside of the cheek, 1 case in the soft palate and one laryngotracheal case.

Results and discussion: The management of adenoid cystic carcinoma remains delicate because of their insidious growth, their neurological tropism and their metastatic potential. These associated elements delay the diagnosis that is often made while those tumors are locally advanced, which can make surgery difficult, and be responsible of a lot of late recurrences. The treatment of these tumors has long relied on exclusive surgery. Cystic adenoid carcinomas were considered radio resistant. Retrospective studies have shown the benefit of treatment combining surgery and radiotherapy, in terms of local control and disease-free survival, compared to surgery or radiotherapy alone.

Conclusion: The essential problem of adenoid cystic carcinoma remains the long-term control of the disease. Our results confirm that the gold-standard treatment should be both surgery and radiotherapy.

Keywords: adenoid cystic carcinoma- atypical localization-surgery.
Submitted : March 5, 2021

Published : March 26, 2021

ISSN: 2593-8339

DOI: $10.24018 /$ ejmed.2021.3.2.752

\section{F. Hadid*}

Ear, Nose and Throat Department, Mohammed VI University Hospital, Marrakesh, Morocco.

(e-mail: fz.hadid@ gmail.com)

M. Hakimi

Ear, Nose and Throat Department, Mohammed VI University Hospital, Marrakesh, Morocco.

O. Oulghoul

Ear, Nose and Throat Department, Mohammed VI University Hospital, Marrakesh, Morocco.

O. Benhommad

Ear, Nose and Throat Department, Mohammed VI University Hospital, Marrakesh, Morocco.

Y. Rochdi

Ear, Nose and Throat Department, Mohammed VI University Hospital, Marrakesh, Morocco.

A. Raji

Ear, Nose and Throat Department,

Mohammed VI University Hospital,

Marrakesh, Morocco.

*Corresponding Author

\section{INTRODUCTION}

Cystic adenoid or cylindroid carcinomas were first described by Robin and Laboulbene in 1852 [1]. The cystic adenoid carcinomas are an infiltrating, very malignant tumor. They are rare and developed usually at the expense of the salivary glands. They represent the main histological type of accessory salivary gland tumors and account for approximately $10-20 \%$ of all salivary gland tumors [2]. They also account for approximately $1 \%$ of all ear, nose and throat cancers, it can be found more rarely in all sites that contain secretory glands (breast, cervix, colon, prostate ...) [3]. Despite a less aggressive histological appearance and satisfactory short-term therapeutic results [4], the management of ear nose and throat cylindromas remain delicate due to their insidious growth, neurological tropism, and metastatic potential. The combination of all these elements delays the diagnosis, which is often made when they are locally advanced, making surgery difficult, and late recurrences frequent. The treatment of these tumors has long relied on exclusive surgery. Until the publications of Stewart and al. and King and al. in the 1970s, cystic adenoid carcinomas were considered radioresistant [5], [6]. Recently, retrospective studies have shown the benefit of treatment combining surgery and radiotherapy, in terms of local control and disease-free survival, compared with surgery or radiotherapy alone [7]-[9].

\section{MATERIALS AND METHODS}

Our work will focus on the analysis of this tumor in some unusual locations. We will be interested in their clinical, paraclinical and anatomopathological characteristics, therapeutic management, and the evolutionary profile of those patients. Our work is a study of 5 cases of cylindromas presenting atypical localizations: 1 case in the external auditory meatus, 1 case in the infra temporal fossa, 1 case in the inner side of the cheek, 1 case in the soft palate and 1 case in the laryngotracheal area. These cases were collected in the ear nose throat and cervicofacial surgery department 
of university hospital Mohamed IV in the city of Marrakesh, our work was based on the study of all medical files.

\section{RESULTS}

\section{A. Case File 1}

Mrs. B. B, 61 years old, with no notable medical or surgical history, was addressed for a left unilateral otalgia evolving for 8 months, without otorrhea, nor hypoacusis or tinnitus, the clinical examination found a polypoid tumor of the left external acoustic meatus in its bony part associated with epidermal debris, completely hiding the tympanic membrane, the contralateral ear was normal, the facial function, also, was normal, the audiogram showed a normal hearing. At the computerized tomography scan of the temporal bone: presence of a polypoid filling of the external auditory canal, completely covering the tympanic membrane, measuring about $18 \mathrm{~mm}$ thick and $8 \mathrm{~mm}$ in diameter with good aeration of the middle ear and mastoid cells, without bone lysis (Fig. 1).

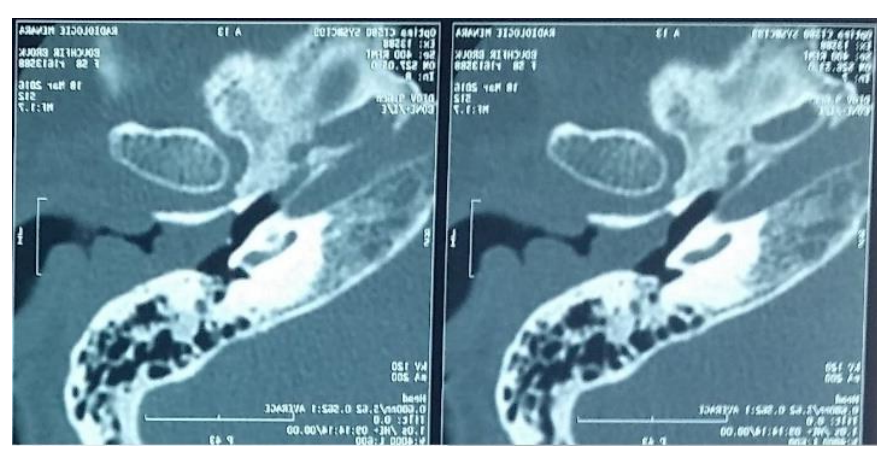

Fig. 1. Computerized tomography scan of the rocks in axial section showing a polypoid filling of the external auditory canal completely covering the tympanic membrane.

An initial biopsy of the polyploid lesion, carried out under local anaesthesia supplemented by immunostaining, concluded that it was a cystic adenoid carcinoma of annexal origin. The surgical management of this case consisted, firstly, of removing the thin skin from the inner side of the thigh and, secondly, removing the supra-malleolar part of the tympanic membrane and the anterior part of the canal as well as the thickened outer area of the canal, milling the walls of the bone canal and reconstructing the tympanic membrane by temporal aponeurosis and positioning the skin graft in contact with the walls of the bone canal, then silastic blades were inserted. Definitive anapath showed healthy limits and classified the case T1N0M0. Post-operative radiotherapy was indicated and the follow-up up to 6 months showed no recurrence of the disease.

\section{B. Case File 2}

Mrs. R. G., aged 59, without any particular pathological history, was admitted to our department for lancinating and insomniating facial pain, not relieved by common painkillers. The pain has been evolving for one year, without nasal obstruction, epistaxis, rhinorrhea, or headaches. The clinical examination revealed a hypoesthesia of the territory of the right maxillary nerve. The rhinocavoscopy was normal. Magnetic resonance imaging (MRI) of the face showed a tumor of the right infra temporal region in hypo signal T1 and hyper signal T2 without endocranial extension (Fig. 2).

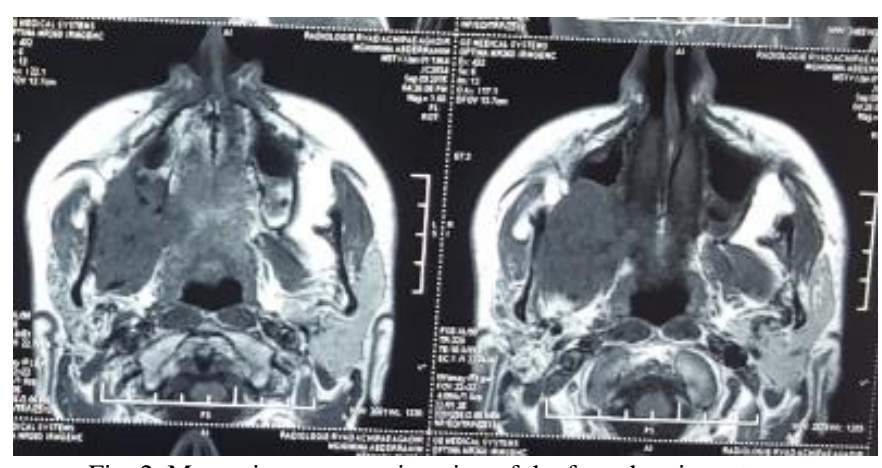

Fig. 2. Magnetic resonance imaging of the face showing a tumour process in the right infra temporal fossa.

A biopsy was done by the Caldwell-Luc method and confirmed a cystic adenoid carcinoma by immunohistochemistry. A thoraco abdominal and pelvic scan was requested as part of the extension check-up but did not reveal any metastases, particularly pulmonary metastasis. The excision was made by a paralateronasal route with a bone flap (Fig. 3). The tumour was adherent to the maxillary nerve and the internal maxillary artery. The excision was considered macroscopically complete with ligation of the internal maxillary artery, sacrifice of the maxillary nerve and milling of the postero-medial wall of the maxillary sinus. The postoperative care of the patient was simple with no bleeding or signs of infection, in particular no rhinorrhea or fever. The anatomopathological study was in favour of a cystic adenoid carcinoma of the infra temporal region with healthy excision margins. The patient was classified as T3NOM0. An adjuvant radiotherapy was carried out by intensity modulation technique (IMRT), delivering a total dose of 70 Gy in 35 sessions of 2 Gy by two arcs in dynamic volumetric arc therapy mode, using 6 MV photons from a rapidarc accelerator. The clinical evolution was favorable. The MRI made out at 3 months from the end of the treatment showed fibrosis scarring, confirmed by biopsy with no signs of recurrence. The patient was seen regularly with no recurrence up to 3 years after his treatment.
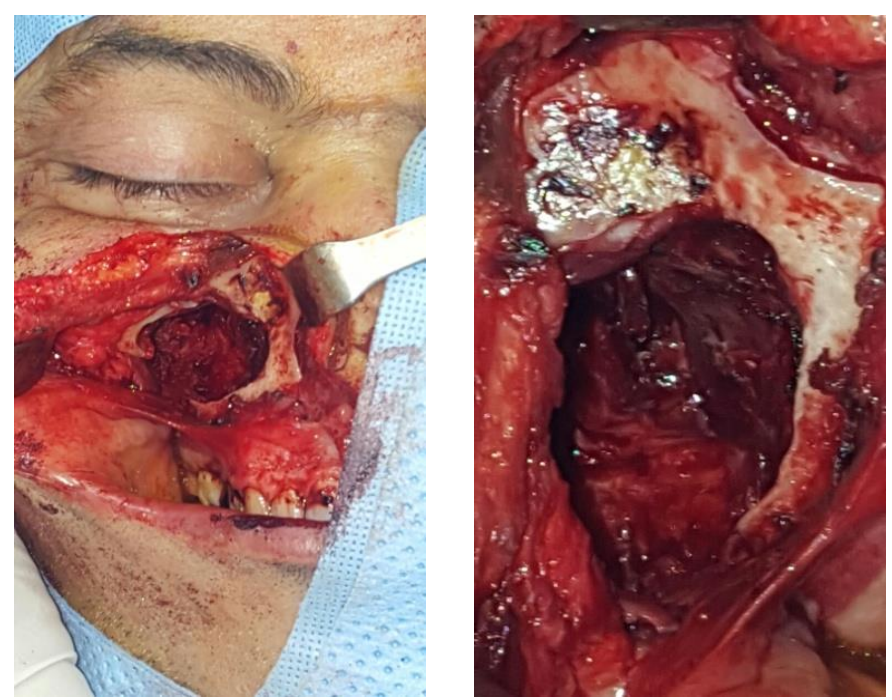

Fig. 3. Peroperative image of a paralateronasal approach with a bone flap. 


\section{Case File 3}

Mrs. J. G, 64 years old with no notable medical history presented with an ulceration of the internal side of the right cheek evolving for 3 years, with a cutaneous extension, without other associated signs (Fig. 4).
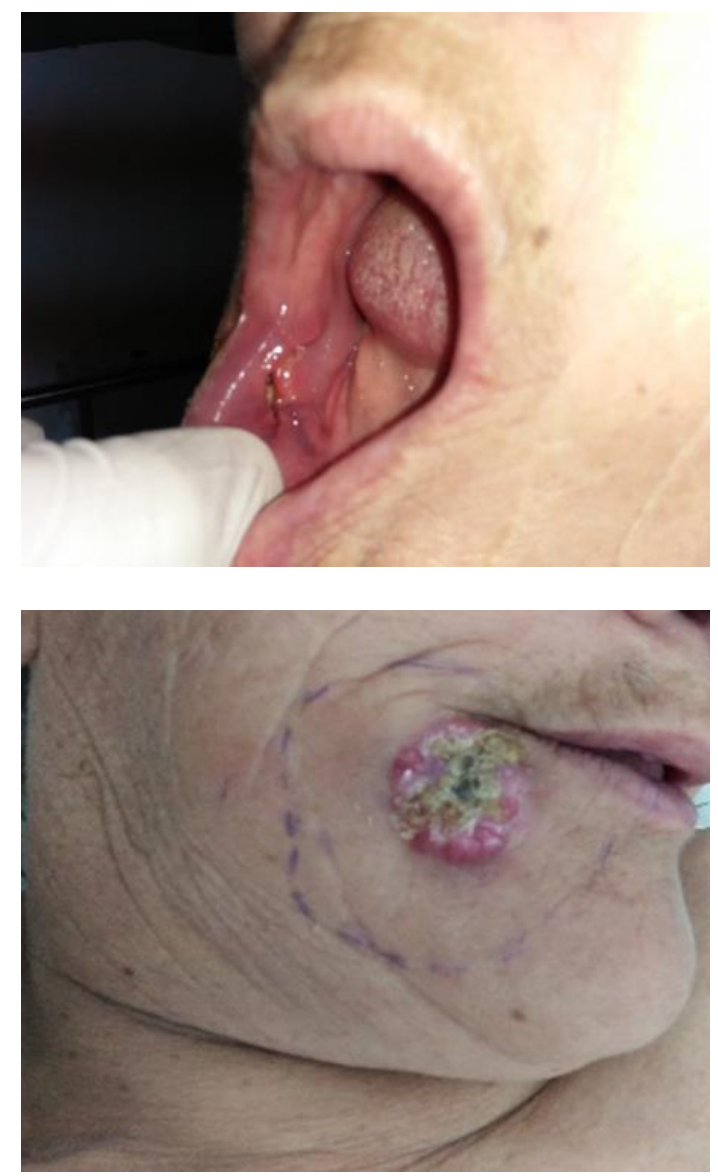

Fig. 4. Image showing the clinical appearance of the tumor on the internal side of the cheek.

The clinical examination showed a $2 \mathrm{~cm}$ painful ulceration of the internal side of the cheek with peripheral induration of $3 \mathrm{~cm}$, with a clinically suspicious submaxillary lymphnode. The CT scan (Fig. 5) showed a lesional process of the soft jugal side of $3.4 \times 3.6 \mathrm{~cm}$ of irregular contours heterogeneous after injection of contrast agent, infiltrating the skin, and in intimate contact with the horizontal branch and the right mandibular angle without bone lysis. The extension check-up done with chest X-ray and abdominal ultrasound was negative.

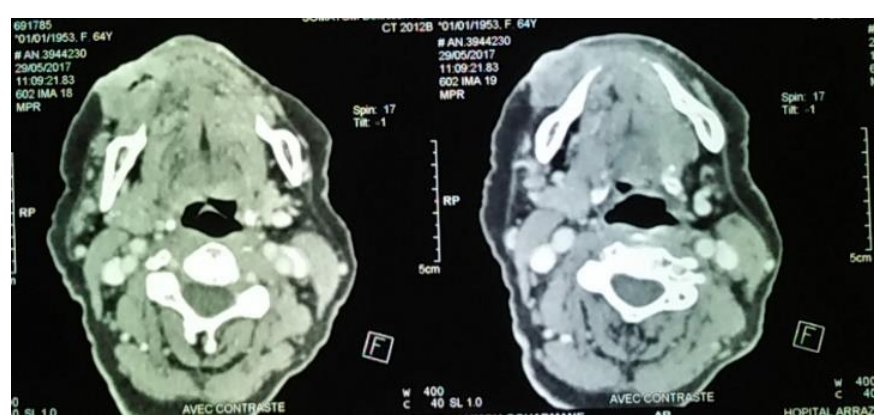

Fig. 5. Computerized tomography scan in axial section showing a straight jugal soft tissue lesion process with irregular contours heterogeneously enhanced after injection of contrast agent infiltrating in front of the skin covering.
A biopsy was done, diagnosing a cylindroma. The surgical management consisted in the first part of a right submaxillectomy after identification and preservation of the XII nerve and lingual nerve, ligation of the facial artery and vein, lymph node dissection of the Ia, Ib, IIa lymphnode groups and the second part was the removal of the jugal tumour with a jugal advancement flap. The postoperative follow-up was simple.

The final anapath concluded in a cystic adenoid carcinoma classified T2N1M0 with satisfactory excision margins. Post-operative radiotherapy was indicated. A 3month follow-up was without incident.

\section{Case File 4}

Mrs. L. F., 46 years old, without any particular pathological history, presented with an intraoral swelling gradually increasing in volume over the last two years, hindering swallowing, without any other associated signs including no bleeding, nor dyspnea. The clinical examination found a mass of the soft palate more developed on the right side, measuring $5 \mathrm{~cm} \times 4 \mathrm{~cm}$, firm, painless, displacing the right anterior pillar with central ulceration (biopsy area) (Fig. 6).

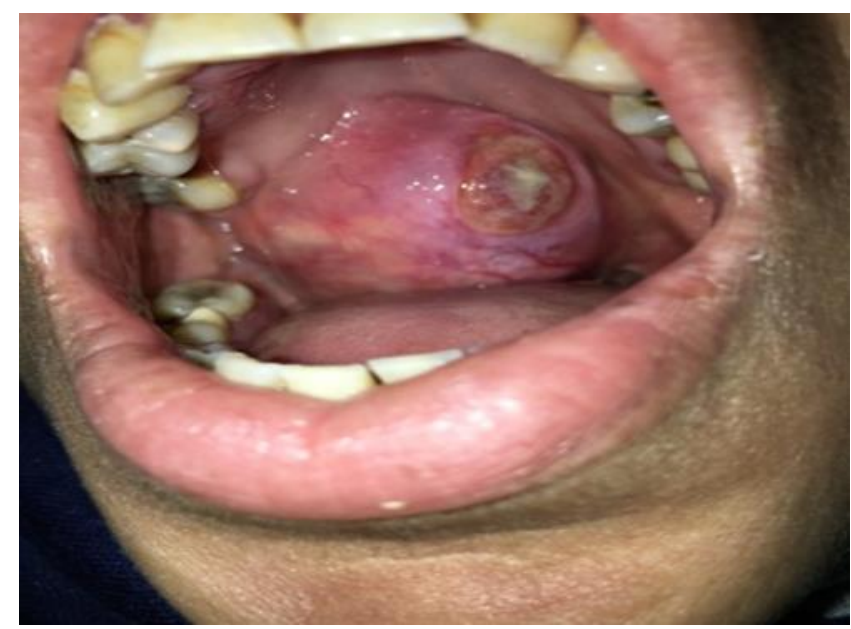

Fig. 6. Image showing the clinical aspect of the palatal tumor mass.

At the cervicofacial computerized tomography scan: well limited heterogeneous oval mass of the right tonsil chamber of $4.93 \times 4.72 \times 4.24 \mathrm{~cm}$, of tissue density with moderate and heterogeneous enhancement without associated calcifications (Fig. 7).

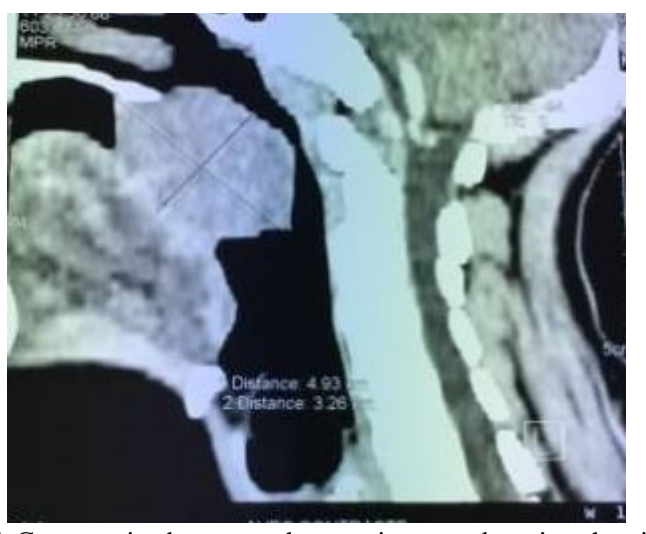

Fig. 7. Computerized tomography scan in coronal section showing a very limited heterogeneous oval mass of the right tonsillar region. 
A biopsy was done with an anatomopathological study completed by immunohistochemistry concluding in a cystic adenoid carcinoma. The extension assessment was negative. Surgery consisted of block endobuccal removal of the tumor (Fig. 8).
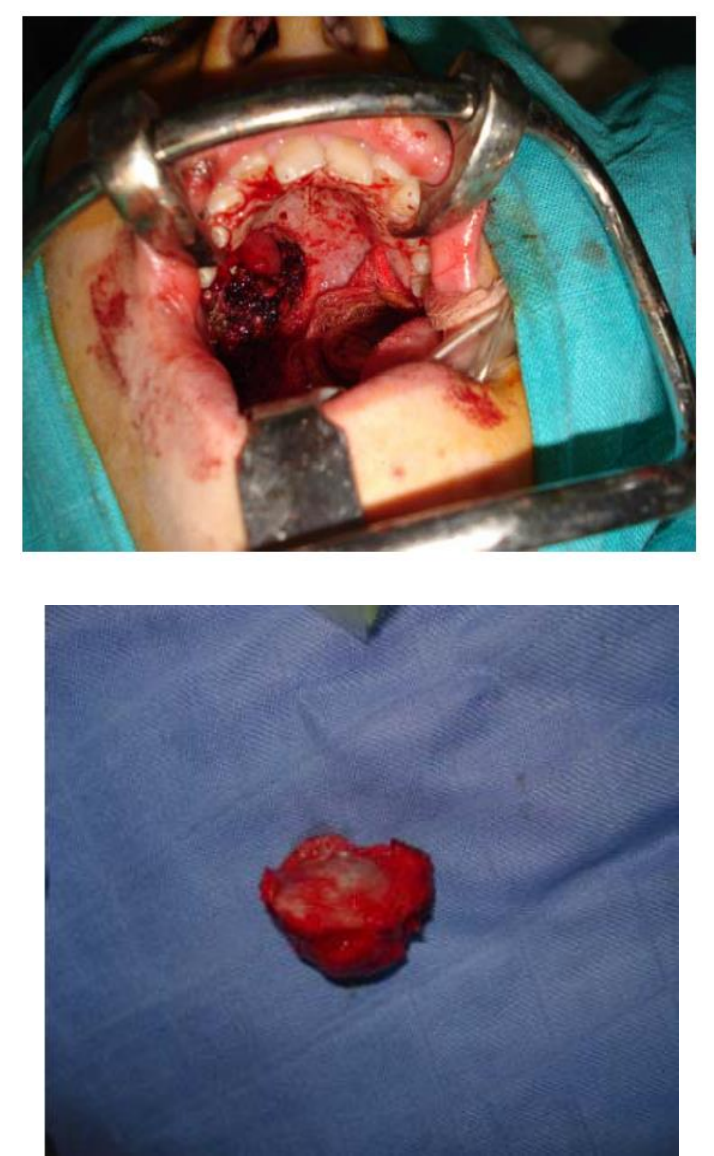

Fig. 8. Intraoperative images showing the monobloc excision of the tumor.

The postoperative follow-up was simple (Fig. 9). Definitive histological examination confirmed the diagnosis of cystic adenoid carcinoma with satisfactory excisional limits, classifying it as T3N0M0, further radiotherapy was not indicated. The follow-up of 16 months showed no signs of recurrence.

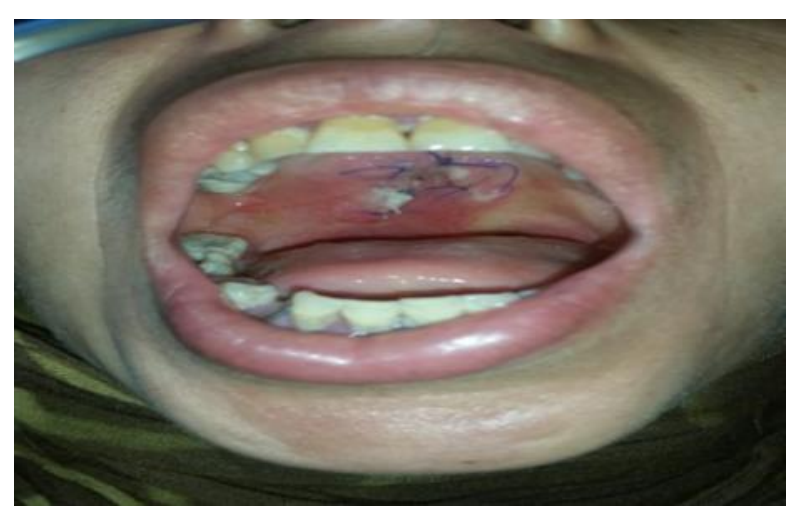

Fig. 9. Post-operative image after total resection of the palatal tumor.

\section{E. Case File 5}

Mrs K. A., 46 years old, was hospitalized in the ENT department for the management of a chronic dysphonia evolving for 2 years associated with a progressively worsening laryngeal dyspnea evolving for 2 months. She had no particular pathological history and was neither a smoker nor an alcoholic. The aggravation of laryngeal dyspnea motivated a tracheotomy. Nasofibroscopy objectified an obstructive subglottis tumor, vocal nodes were of normal aspect and mobility, the upper glottic floor was free. The cervical lymph node areas were free. We performed a cervical scan that showed a process of the post laryngeal wall of the subglottic floor of the larynx at the C6 - C7 level, measuring $32 \times 31 \mathrm{~mm}$ totally obstructive, spontaneously hypodense, heterogeneously enhanced after injection of contrast agent responsible of posterior cricoid lysis, moreover it infiltrated the $\mathrm{C} 6$ and $\mathrm{C} 7$ prevertebral muscles without detectable bone lysis, laterally it came into intimate contact with the inner faces of the thyroid lobes with loss of the fat separation interface, infracentimetric jugulocarotid nodes (Fig. 10). The endoscopic examination of the larynx under general anesthesia also allowed to visualize totally obstructive subglottic tumor. Normallooking vocal cords, base of tongue, mouth of esophagus, free piriform sinus and to perform a biopsy of the mass. The results of the anatomopathological examination of the biopsy with histochemical immune study showed a cystic adenoid carcinoma of the larynx. We classified the tumor as T4N0M0. He also had a negative extension assessment. Planned for total laryngectomy associated with bilateral functional lymphadecectomy and total thyroidectomy. The patient refused the operation and was lost to view.

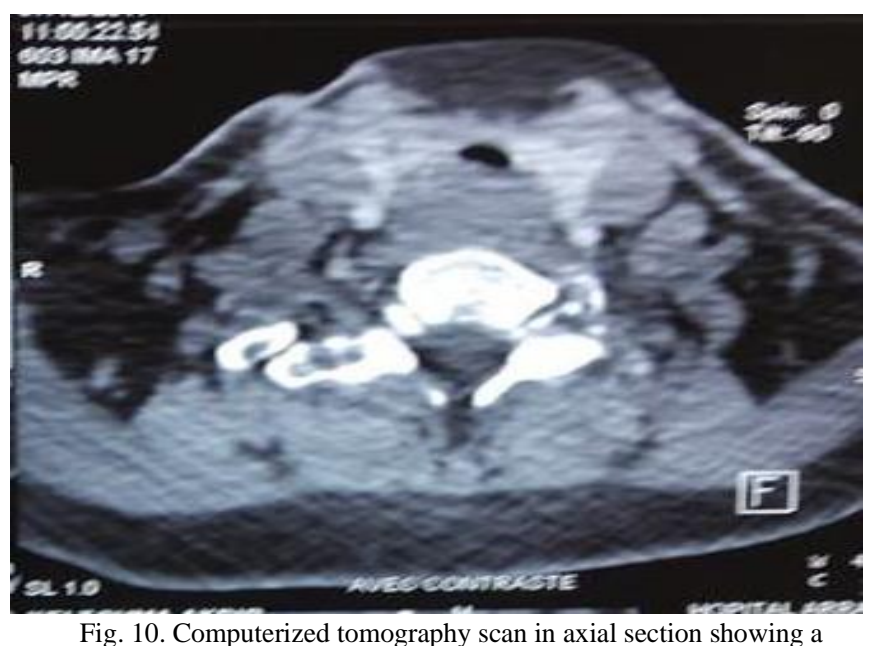

Fig. 10. Computerized tomography scan in axial section showing a lesioned process of the post laryngeal wall of the subglottic stage, at the level of C6 C7 totally obstructive, spontaneously hypodense, raised heterogeneously after injection of contrast agent.

\section{DISCUSSION}

Cystic adenoid carcinomas, also known as Cylindromas, are mainly found in the salivary glands, respiratory mucosa and lacrimal glands. Rarely, in the breast, cervix, skin, Bartholin and Cowper glands are described. The histogenesis of this tumor is still under discussion. It is currently accepted that adenoid cystic carcinoma is derived from modified apocrine cells [2], [5]. In our serie, all our cases were female $(100 \%)$; the same thing was dicribed in multiple studies where there is a predominance of the female sex, as shown for example in the series of M. Meyers et al. a prospective multicenter study of 95 cases, where $64.2 \%$ of 
patients were female [11], and also the series of T. Duberge and al., a multicenter retrospective study of 169 cases, a sex ratio of 1 male to 1.5 female was found [10]. The median age of diagnosis in our study was 55.2 years (46-64), which is completely concordant with the literature with an age of diagnosis that is in the sixth decade for the majority of authors. However, there are some cases that were diagnosed before the age of 20 and after the age 80 [12], [13].

A study conducted on 2010, concerning 99 patients with cystic adenoid carcinoma, reported that the female sex was pejoratively related to high rates of recidivism suggesting a hormonal influence [12], which can explain the high incidence of this pathology in female patients.

According to T. Duberge and al [10], the most frequent tumor site was the parotid (48 patients, $28.4 \%$ ), then palate (11 cases, 6.5\%), external auditory canal (5 patients, $3 \%$ ) and finally trachea ( 2 patients, $1.2 \%$ ). In a prospective multi-center study of 95 cases, M. Meeyres and al. [11] found that the parotid was the most common site of cystic adenoid carcinoma in $25 \%$ of cases, the trachea in $2 \%$, the subglottis in $2 \%$, the oral floor in $2 \%$, and the external auditory canal in $1 \%$. The histological diagnosis of these tumors requires a deep and wide enough biopsy in order to properly characterize the tumor [3], [6], [7]. Immunohistochemistry techniques are used to optimize the diagnosis of cystic adenoid carcinoma [6]. There is no consensus concerning the realization of imaging for the extension assessment. The local assessment uses the computerized tomography scan, more or less associated with a magnetic resonance imaging. The remote assessment requires a chest X-ray or a chest scan and an abdominal ultrasound [10]. Surgery is the gold-standard treatment for cystic adenoid carcinoma regardless of their initial location with the aim of achieving a complete removal of the tumor (one-piece removol, block removal) [14]. Our series confirms the importance of surgery in the therapeutic strategy. The management was decided to be surgical in all our patients, 4/5 of our patients were operated on without any lymphadectomy; with flap reconstruction in two cases. Only one patient who was operated on at the primary tumor site had surgery on the lymph node areas. This exact therapeutic strategy is reported in the literature: lymph node removal should only be performed in high grade, lymphaticrich areas. The majority of unilateral scrapings (complete or partial) were performed in the main salivary gland sites (parotid and submandibular glands) (regardless of the Tstage); bilateral scrapings were mainly performed in the pharyngolaryngeal sites (with midline involvement). There is no operative indication for lymph node dissection in cylindromas of the facial regions (regardless of the stage $\mathrm{T}$ ) [15], [16].

Post-operative radiotherapy is also an important part of the management. In our series, the combination of surgery and postoperative radiotherapy has been recommended. The majority of data in the literature have reported the benefits of adjuvant radiotherapy in terms of local control: Miglianico and al. showed in 102 patients with cystic adenoid carcinoma of the head and neck a local control rate at 5 years of $44 \%$ for patients treated by surgery alone, compared to $77.8 \%$ for patients treated by surgery followed by radiotherapy $(\mathrm{p} \leq 0.01)[17]$.
Diagnosis of cystic adenoid carcinoma of the external acoustic meatus is often delayed. Approximately 150 cases have been described in the literature. The largest series of cystic adenoid carcinoma of the external acoustic meatus is that of Perzin and al. reporting only 16 cases [18]. This tumor is insidious and slow growing [18], [19]. The average interval between the onset symptoms and diagnosis could be several years. This diagnostic delay is mainly due to the non-specificity of the clinical signs. The most common symptom in literature is resistant to treatment otalgia. This symptom is almost constant and is thought to be related to tumor invasion of the perineurium of adjacent sensory nerves or of the periosteum and the adjacent perichondrium. Radiological signs are discreet and rarely suggestive of malignancy [20], [19]. Cystic adenoid carcinomas of the external acoustic meatus require block resection of the latter with a petromastoid recess enlarged on demand to the surrounding structures, up to subtotal petrectomy. Parotidectomy is essential, since the parotid gland can be the site of recurrences and local invasion by tumour dissemination along the perineurium of the facial nerve [18]. Homolateral cervical lymph node dissection is discussed by some authors as it would not change the locoregional prognosis due to the low lymphophilia of cystic adenoid carcinoma of the external auditory meatus [20]. Selective lymph node dissection can be discussed on a case-by-case basis, depending on the extent of the tumor. In our case, the tumor was at an early stage T1NOM0, with no anterior extension (zygomatic bone, temporomandibular joint), no bone lysis, and no extension to the middle ear. We decided to remove in the first stage a sample of the thin skin on the inner side of the thigh that was used in the second stage ; after the removal of the supra-mallear part of the tympanic membrane and the anterior part of the duct and the thickened external area of the duct, Milling of the walls of the bone duct and reconstruction of the tympanic membrane by temporal aponeurosis; and used it in contact with the walls of the bone duct to avoid a stenosis of the external acoustic meatus .

Complementary radiotherapy is not indicated for large excisional procedures with healthy margins [18]. Adjuvant chemotherapy is not mandatory; it is decided based on the high risk of recurrence. The prognosis for cystic adenoid carcinoma of the external acoustic meatus depends on the margins of excision, bone invasion, initial parotid involvement, and nerve invasion [20], [18]. The localization of cystic adenoid carcinoma of the external acoustic meatus increases the aggressiveness of this tumor. The risk of recurrence due to its metastatic power is estimated at $30 \%$ according to some authors [21]. Secondary localizations are mainly pulmonary, but also bony, renal and cerebral [22].

Primary cystic adenoid carcinomas of the infratemporal region are exceptional According to Conley [23], tumors that develop at the expense of this region may originate either from the various constituents of this region, from neighboring structures or constitute metastasis [23, 25, 26]. Primitive cystic adenoid carcinoma of the infratemporal region is characterized by an insidious evolution, which is the reason of diagnosis delay, especially in this clinically silent region [23]-[25], [28], and by their neurological tropism [24]. Computed tomography and magnetic 
resonance imaging currently allow a complete exploration of this region [23], [24], [27]. Transantral or transoral biopsy allows histological confirmation and guides the therapeutic approach [23], [24].

The therapeutic management of cystic adenoid carcinoma of the infratemporal fossa is comparable to other localizations: it mainly relies on surgery even in advanced cases classified as T4 [24], [26]. Survival remains better in patients treated with surgery compared to patients treated with chemoradiotherapy alone [24].

Cystic adenoid carcinomas account for $5-10 \%$ of tracheal cancers. Cystic adenoid carcinomas are the second most common type, after squamous cell carcinomas. Laryngeal involvement, on the other hand, is exceptional (less than $0.25 \%$ of laryngeal tumors) due to the rare occurrence of accessory salivary glands at this level that have a preferential subglottic distribution [25]. These are lowgrade, slow-growing tumors, with the first clinical sign sometimes dating back several months or even years. Classically, the local extension is submucosal with a longitudinal and transverse progression, with invasion of the peri-laryngeal structures in the advanced forms, notably due to an often-late initial diagnosis. Extensions along the perinerve sheaths (recurrent nerve) are very often described but not specific. The presence of lymph node metastasis is possible, but uncommon (10 to $15 \%$ ). Remote metastasis (15 to $40 \%$ ) occurs, in order of preference, in the lungs, bones, brain, and more rarely in the liver.

In cystic adenoid carcinoma of the oral cavity, the palate is the site most often involved [30], the tongue being second; other rarer sites are the oral floor, the lips, and the inner side of the cheek [31]. A Chinese study of 45 patients with cystic adenoid carcinoma of the palate, due to an early diagnosis, were simply treated with a surgical resection with good excision margins, it was largely enough and showed no long-term recurrence [30].

\section{CONCLUSION}

Adenoid cystic carcinoma is a malignant tumor affecting both major and minor salivary glands, but also lacrimal glands, and serous glands of the respiratory mucosa. Its natural history is known for its prolonged evolution, its tendency to recurrence and late distant metastases, mainly pulmonary. It is a fearsome tumor due to its extension via the perinervous sheaths. The efficacy of different management policies on long-term survival remains difficult to predict. A complete surgical resection enlarged if possible, with homolateral lymph node dissection in the presence of lymph nodes. Post-operative radiotherapy is indicated in high-grade tumors in stages II to IV and in lowgrade tumors in stages III and IV; it is not indicated for fully resected low-grade tumors in stages I and II. The search for targeted therapies for metastatic cases or relapsing patients is a future prospect. It involves studies, particularly genomic studies, that can be carried out on samples and stored in tumor libraries.

\section{CONFLICT OF INTEREST}

The authors do not declare any conflict of interest.

\section{CONTRIBUTIONS OF THE AUTHORS}

All the authors contributed to this work.

All authors also declare that they have read and approved the final version of the manuscript.

\section{REFERENCES}

[1] Tauxe WN, Mc DJ, Devine KD. A century of cylindromas. Short review and report of 27 adenoid cystic carcinomas arising in the upper respiratory passages. Arch Otolaryngol 1962;75:364-76.

[2] Spiro RH, Huvos AG, Strong EW. Adenoid cystic carcinoma of salivary origin. A clinicopathologic study of 242 cases. Am J Surg 1974;128:512-20.

[3] Spiro RH. Management of malignant tumors of the salivary glands. Oncology (Williston Park) 1998;12:671-80 [discussion 83].

[4] Deangelis AF, Tsui A, Wiesenfeld D, Chandu A. Outcomes of patients with adenoid cystic carcinoma of the minor salivary glands. Int J Oral Maxillofac Surg 2011;40:710-4.

[5] Stewart JG, Jackson AW, Chew MK. The role of radiotherapy in the management of malignant tumors of the salivary glands. AJR Am J Roentgenol 1968;102:100-8.

[6] King JJ, Fletcher GH. Malignant tumors of the major salivary glands. Radiology 1971;100:381-4.

[7] Mendenhall WM, Morris CG, Amdur RJ, Werning JW, Hinerman RW, Villaret DB. Radiotherapy alone or combined with surgery for adenoid cystic carcinoma of the head and neck. Head Neck 2004;26:154-62.

[8] Miglianico L, Eschwège F, Marandas P, Wibault P. Cervico-facial adenoid cystic carcinoma: study of 102 cases. Influence of radiation therapy. Int J Radiat Oncol Biol Phys 1987; 13:673-8.

[9] Vikram B, Strong EW, Shah JP, Spiro RH. Radiation therapy in adenoid cystic carcinoma. Int J Radiat Oncol Biol Phys 1984; 10: 221-3.

[10] T. Dubergé, K. Bénézery, M. Resbeut, D. Azria, M. Minsat, S. Ellis, E. Teissier, A. Zaccariotto, C. Champetier, D. Cowen Adenoid cystic carcinoma of the head and neck: A retrospective series of 169 cases.

[11] M. Meyers, B. Granger, P. Herman, F. Janot, R. Garrel, N. Fakhry, G Poissonnet, B. Baujat, et les membres du REFCOR1 Les carcinomes adénoïdes kystiques ORL: une étude multicentrique prospective REFCOR de 95 cas.

[12] Oplatek A, Ozer E, Agrawal A, et al. Patterns of recurrence and survival of head and neck adenoid cystic carcinoma after definitive resection. Laryngoscope 2010; 120: 65-70.

[13] Wiseman SM, Popat SR, Rigual NR, et al. Adenoid cystic carcinoma of the para-nasal sinuses or nasal cavity: a 40-year review of 35 cases. Ear Nose Throat J2002; 81: 510-4.

[14] Garden AS, Weber RS, Morrison WH, et al. The influence of positive margins and nerve invasion in adenoid cystic carcinoma of the head and neck treated with surgery and radiation. Int J Radiat Oncol Biol Phys 1995; 32: 619-26.

[15] Bosch A, Brandenburg JH, Gilchrist KW. Lymph node metastases in adenoid cystic carcinoma of the submaxillary gland. Cancer 1980; 45: 2872-7.

[16] Min R, Siyi L, Wenjun Y, et al. Salivary gland adenoid cystic carcinoma with cervical lymph node metastasis: a preliminary study of 62 cases. Int J Oral Maxillofac Surg 2012; 41: 952-7.

[17] Mendenhall WM, Morris CG, Amdur RJ, Werning JW, Hinerman RW, Villaret DB. Radiotherapy alone or combined with surgery for adenoid cystic carcinoma of the head and neck. Head Neck 2004;26:154-62.

[18] Perzin KH, Gullane P, Conley J. Adenoid cystic carcinoma involving the external auditory canal. A clinicopathologic study of 16 cases. Cancer 1982; 50: 2873-83.

[19] Fenniche S, Haouet S, Mdimagh H, et al. Les tumeurs des glandes cérumineuses. Ann Pathol1995;15:147-9.

[20] Dehesdin D, Andrieu-Guitrancourt J, Hemet J, Cauchois P, Marie JP Vinel V. Tumeurs glandulaires du conduit auditif externe. Ann Otolaryngol Chir Cervicofac1993;110:70-4.

[21] Wassef M, Thomas V, Deffrennes D, Lacau Saint-Guily J. Les carcinomes adénoïdes kystiques cutanés primitifs. Ann Pathol 1995; 15: $150-5$. 
[22] Conlin P, Mira JL, Graham S, Kaye KS, Cordero J. Ceruminous gland adenoid cystic carcinoma with controlateral metastasis to the brain. Arch Pathol Lab Med 2002; 126: 87-9.

[23] Rammohan Tiwar et al. Tumors of the Infratemporal Fossa Skull Base. Skull base surgery. 2000; 10(1): 1-9.

[24] Dubergé $T$ et al. Adenoid cystic carcinoma of the head and neck: a retrospective series of 169 cases. Cancer Radiother. 2012 Jul-Aug; 16(4):247-56.

[25] Givi et al. Outcome of resection of Infratemporal fossa tumors. Head Neck. 2013 Nov; 35(11):1567-72.

[26] Iannetti $G$ et al. Infra temporal fossa surgery for malignant diseases. Acta Neurochir (Wien) 1996; 138: 658-71.

[27] Ohue S. Preauricular transzygomatic anterior infratemporal fossa approach for tumors in or around Infratemporal fossa lesions. Acta Neurochir (Wien). 1996;138(6):658-71.

[28] Colmenero C1, Perez Alvarez M, Alonso A. Adenoid cystic carcinoma of the infra spheno-temporal fossa. Latero-facial resection combined with multiple osteotomies. J Craniomaxillofac Surg. 1991 Jul; 19(5):212-6.

[29] Jacquier, V Vidal, JM Thomassin, JM Bartoli, G Moulin, Chinese Journal of Cancer, 2004, 26 (08): 485-489.

[30] Maciel Santos MES, Ibrahim D, Neto JC, Da Silva JC, Da Silva UH, Sobral APV. Carcinoma adenóide cístico: relato de caso RevCirTraumatol Buco-Maxilo-Fac. 2005; 5:49- 54. PubMed Google Scholar. 